\title{
Ethnobotanical Study of Rice Growing Process in Northeastern, Thailand
}

\author{
Piya Mokkamul
}

Pre-Publication Review Draft

\section{Photo Essay}

\begin{abstract}
Thailand is a developing country that relies heavily on rice agriculture. Photographic techniques are an easy and efficient method for studying human activities, culture, traditions, ethnobotany, and ethnoeconomy. In this paper, photos are used to provide an ethnobotanical description of the rice growing process of rice farmers in Northeast Thailand, from preparing the rice fields to harvesting the rice.
\end{abstract}

"One picture can speak a thousand words."

\section{Introduction}

Thailand is a developing country that relies heavily on agriculture. Seventy percent of Thailand's 64 million people are farmers and the majority cultivate rice. Until ten years ago, rice was the most economically valuable export of Thailand. Since then income from tourism has taken the lead.

Most of rice farmers in northeastern Thailand (Isaan) use growing methods that they learned from their ancestors. They usually grow rice once a year, beginning in the rainy season (June-August) and harvest at the beginning of the cold/dry season (November-December). This this is known as na pee. Farmers rarely grow rice two times a year but when they do, they rely on water collected in dams and grow the second crop during the dry season. This second crop is know as na plang. There are two types of rice field in the northeast Thailand which are determined by the method used to grow the rice. Na daam refers to a rice field which is grown from rice seedlings. $\mathrm{Na}$ waan refers to a rice field which is grown by scattering rice seeds throughout the harrowed field blocks. Most rice fields in Thailand are na daam. Isaan rice farmers usually grow glutinous rice (Oryza sativa L. var. glutinosa (Lour.) Körn) for personal consumption and non-glutinous jasmine rice (Oryza sativa L. var. sativa) for sale because jasmine rice can be sold for a greater price. The method of growing rice among rice farmers in northeastern, Thailand using the na daam process (Worakawin 2004) is outlined below.

1. When the level of the water in the rice paddies is sufficiently high enough to kill other weeds and produce rice seedlings at that time, the field is plowed for the first time.

2. A few rice paddies are sown with rice plants. The remaining paddies are plowed for a second time when the rice seedlings are two months old.

3. The paddies that were recently plowed are harrowed to flatten the surface of the soil. The rice seedlings that are being grown in the few separate paddies are pulled out, tied into bunches, and the upper part of their leaves are cut to reduce plant transpiration.

4. Four to five rice seedling stems are clustered together and planted about $30 \mathrm{~cm}$ apart in the harrowed rice paddies. This method consists of clasping the cluster of seedlings in a fist and pushing the seedlings into the soil with the thumb. This method, called daam, gives the rice cultivation process its name.

\section{Correspondence}

Piya Mokkamul, Department of Biology, Faculty of Science, Khon Kaen University, Khon Kaen, 40002, THAILAND.

piyaatg@yahoo.com

Ethnobotany Research \& Applications 4:213-222 (2006) 
5. The rice plants are cut down when the fruits are ripe, collected, and threshed to remove the rice seeds.

6. The rice is then stored in a barn to await sale.

There are many methods that can be used to study the cultivation process of rice, for example interviews with rice farmers and rice markets surveys. Today, photos are a necessary tool that can be used by everyone in most areas of science research. Photos can be very helpful in ethnobotanical studies (Vogl-Lukasser \& Vogl 2005) by continuing to produce good information long after the field data collection process. Photos can assist in locating plants for collection, species identification, documentation of traditionally used tools, recording traditional events and agricultural processes. Pictures can be used to describe research methods and results in publications in order to make them more attractive and help the audience to visualize the topic and attract interest.

Cameras are used by people of all ages and occupations throughout much of the world. Today, almost every household in Thailand owns at least one camera to record pictures of special events, beautiful landscape scenes, and human activities. People in Thailand have become accustomed to using cameras, both taking pictures and having their pictures taken.

Cameras have improved over the years in functional properties and technology. The bulky camera of the past changed into a more compact film camera with manual focusing lenses. Modern cameras are greater ability digital cameras. These cameras have many high technology functions (rapid auto focusing lenses/system, auto flash, high accuracy light metering, high quality photo sensors, etc.) that are often packaged into very light small bodies, that can be used by all levels of photographers. Everyone has the potential to shoot a very "good" picture by just pressing one shutter button. Professional photographers can control and apply camera functions and accessories by combining personal ideas, artistic angles, photo exposure, shutter speed, different lenses, depth of field, as well as many other variables. By becoming proficient in all of these skills, the professional photographer has the ability to shoot a more "perfect" picture with more meaning than those of an amateur photographer.

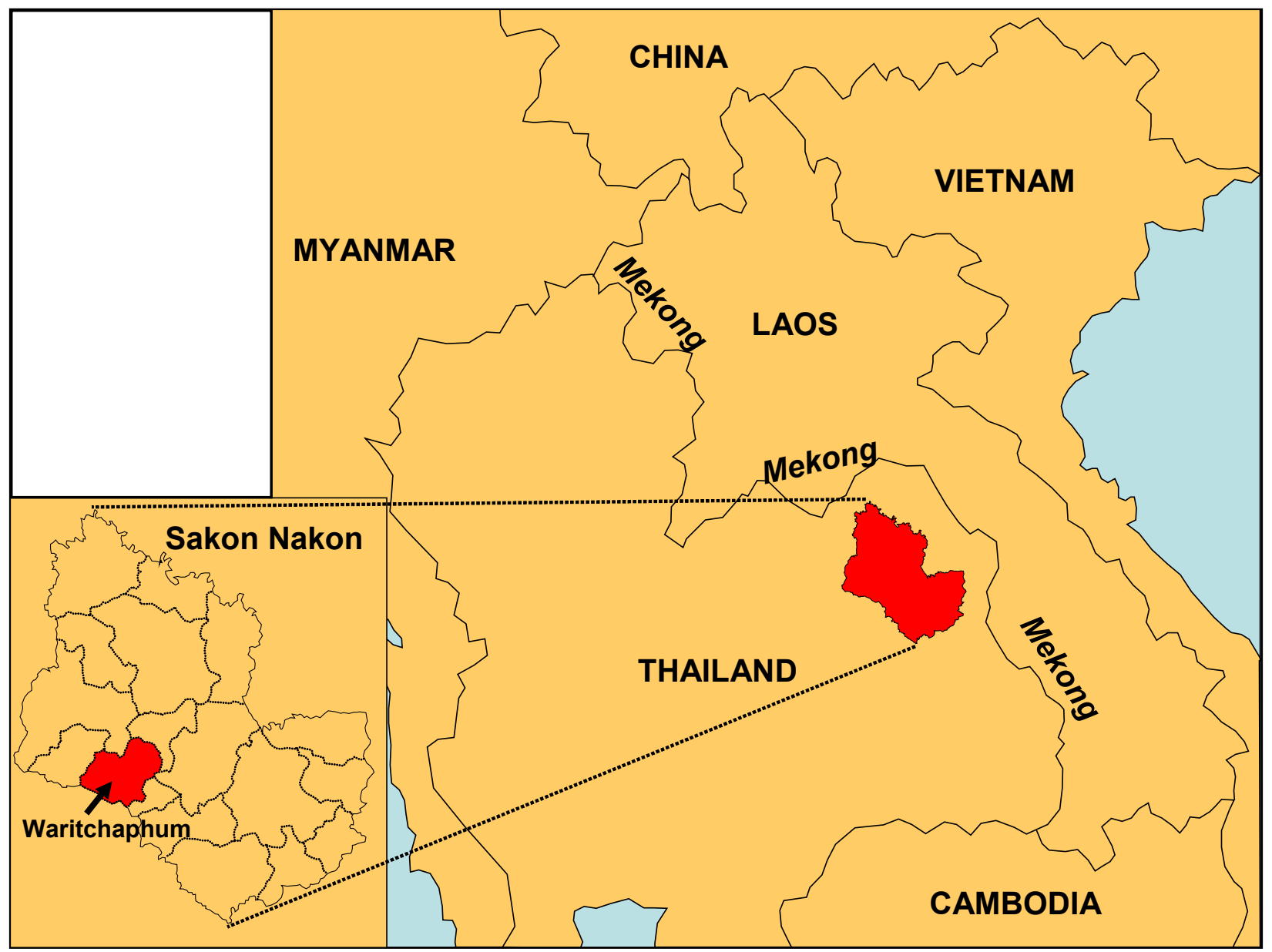

Figure 1. One of the primary photography locations in Tambon Kham Bo, Waritchaphum, Sakon Nakon, Thailand. 


\section{Mokkamul - Ethnobotanical Study of Rice Growing in Northeastern, Thailand 215}

\section{Methodology}

The author took all the photos from 2002 to 2006 using either:

1. a Canon EOS 10D digital SLR camera, and a 16-35 $\mathrm{mm}$ zoom lens, a 28-105 mm zoom lens, a $100 \mathrm{~mm}$ macro lens, with $550 \mathrm{EX}$ flash, or

2. an Olympus C3030 compact camera with a polarization filter.

Thirteen villages in northeast, Thailand (Figure 1) in different seasons were visited to photograph the community activities involved with the rice growing process. Some rice field landscape scenes were photographed by using a super wide angle zoom lenses of $16-35 \mathrm{~mm}$ and standard zoom lenses of 28-105 $\mathrm{mm}$ because the photo's depth of field is easily manipulated. An f-stop range of $f 4-f 8$ for super wide angle lenses (at $16 \mathrm{~mm}$ ) and $\mathrm{f} 8-\mathrm{f} 11$ for a standard zoom lenses (at $28 \mathrm{~mm}$ ) was used. When using a tripod, the f-stop number could also be set at $f 16$, the maximum f-stop value. For shooting human activities and portrait pictures a standard zoom lens of 28-105 mm with a wide angle range zoom was used, and the focal length of this lens was used to shoot candid or snap shot photos by zooming to tele-photo focal length. For portrait shots, a standard lens of $50 \mathrm{~mm}, 85 \mathrm{~mm}$ or a macro lens of 100 $\mathrm{mm}$ was used, for example, to focus on people's eyes because you can see their emotions in their eyes. High shutter speeds was also used to catch people in motion.

It was important to develop good relationships with the local people first and ask for their permission to take some photos of their rice field, rice growing equipment and rice plants in the fields. Farmers were given gifts and copies of the photos in exchange for participating in the research.

\section{Results}

Photos of the process of rice farming are presented in Figures 2-13 following the steps outlined above.

1. Plowing (Figure 2).

2. Nursery paddy (Figures 3-4).

3. Planting paddies (Figure 5).

4. Planting clusters of seedlings using the daam method (Figures 5-6).

5. Rice field growing (Figure 7), grown (Figure 8), being harvested (Figures 9-13), and threshed ((Figure 10).

6. Storage of rice in a barn (Figure 11) and prepared to eat (Figure 12).

\section{Conclusion}

Photos are useful and necessary for all types of science research and most particularly for ethnobotany research that focuses on the activities and relationships between people and plants. Academically, photos can be used to facilitate reports and publications, and presentations of the research. They can also help to improve the quality of research results by creating closer ties between the researchers and the local people. Photos are a good medium for reaching the general public to improve their attitude towards the value of community traditions, culture and local wisdom. Photos are important for the local, regional, national and international impact of research.

\section{Acknowledgements}

The author wishes to thank Will McClatchey for his editorial advice, technical assistance and publication support, Assistant Professor Arunrat Chaveerach, Miss Runglawan Sudmoon and Mr. Tawatchai Thanee for their kind and generous encouragement, and Mr. Khomsan Khamphitchu for his technical assistant.

\section{Literature Cited}

Vogl-Lukasser, B. \& C.R. Vogl. 2005. Ethnobotanical Research in Homegardens of Small Farmers in the Alpine Region of Osttirol (Austria): Photo Essay. Ethnobotany Research \& Applications 3:079-081.

Worakawin, K. 2004. Isaan Muea Wun Waan (in Thai). Dr. Koh Worakawin foundation for reading support, Bangkok. 


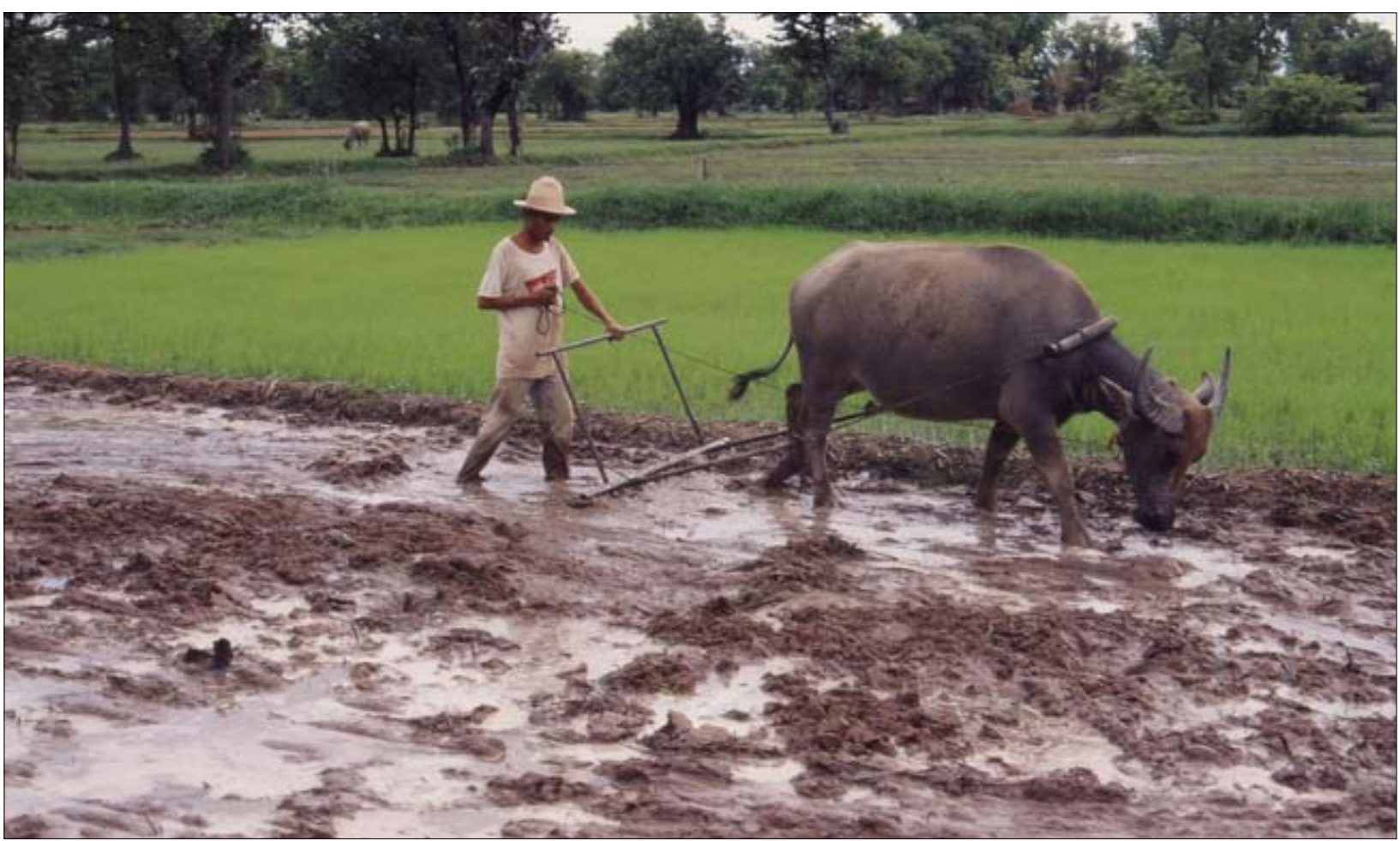

Figure 2. A farmer plowing a paddy field for the first time.

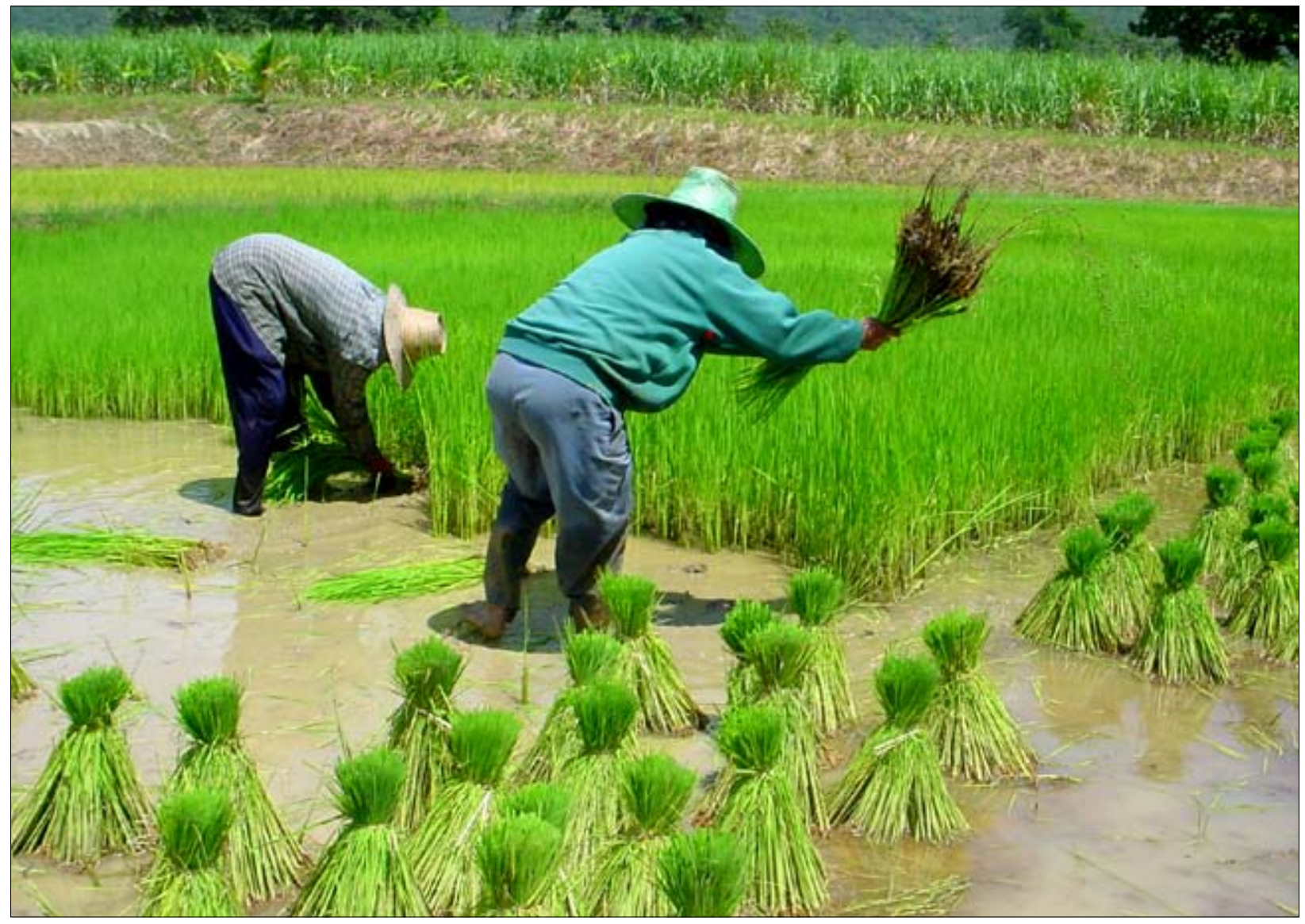

Figure 3. Pulling rice seedlings and removing some soil from their roots by hitting on the feet. 


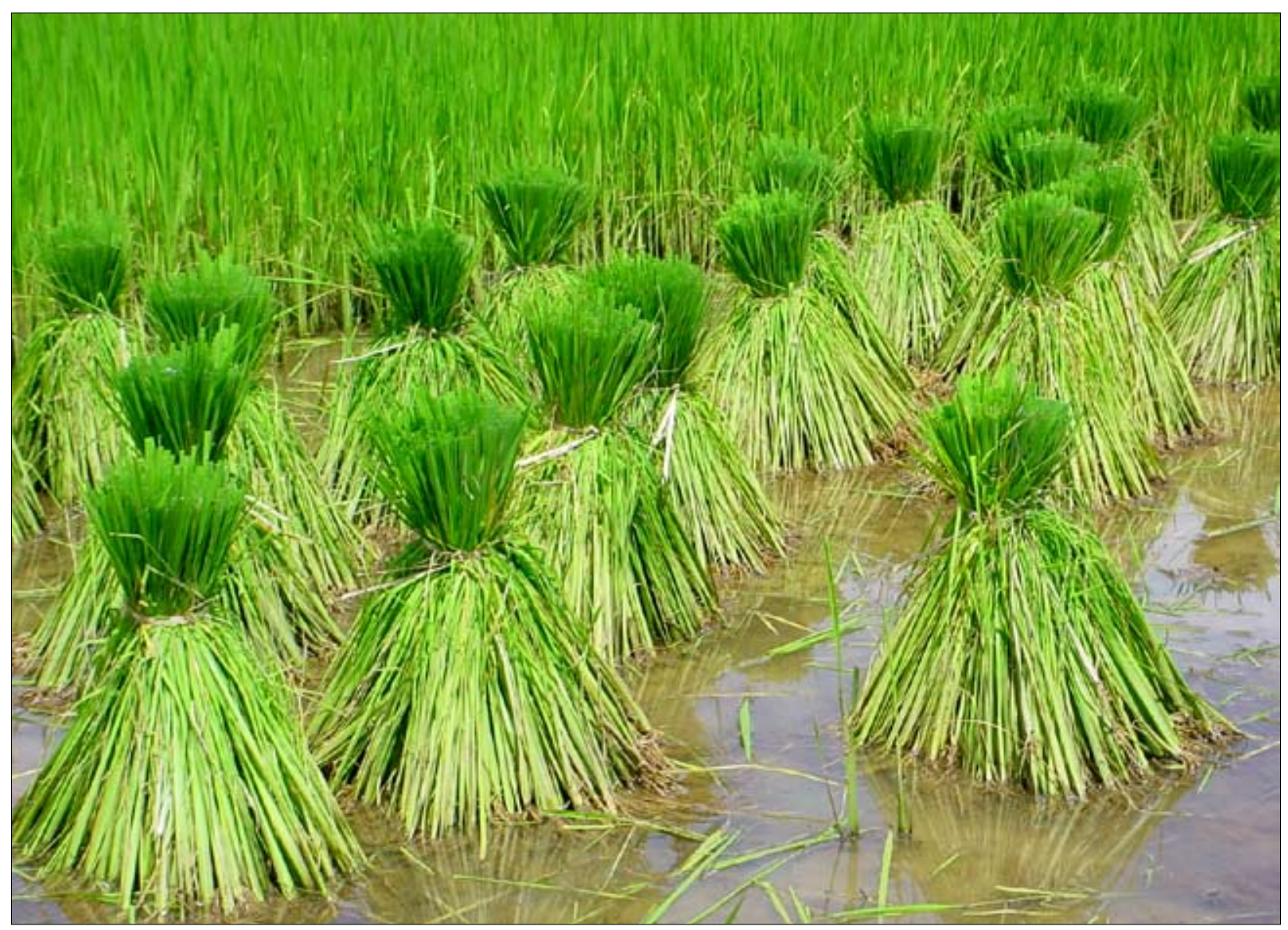

Figure 4. Bunches of rice seedlings after cutting upper part of leaves.

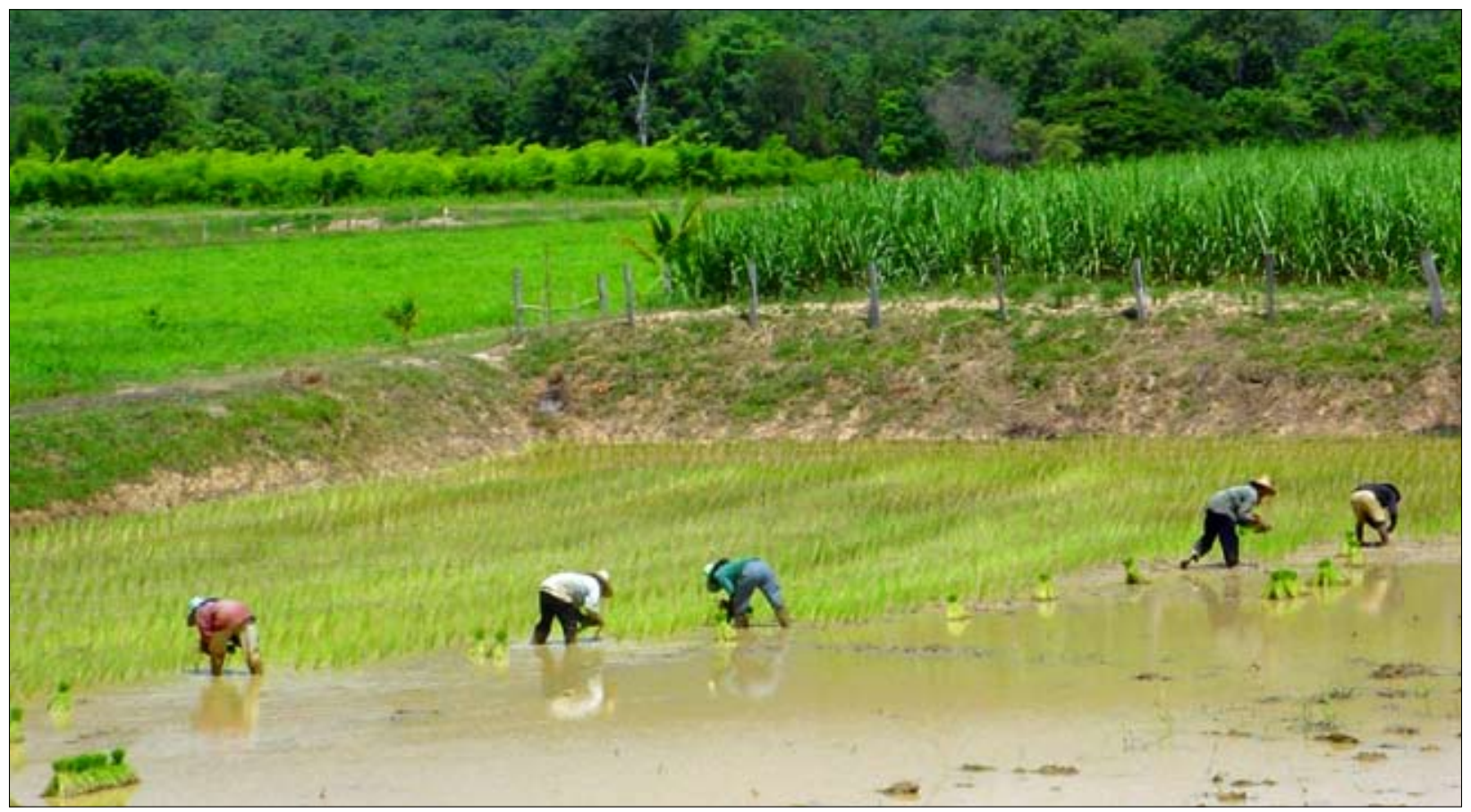

Figure 5. Farmers planting one by one many clusters of rice seedlings into a harrowed and flattened paddy field (na daam). 


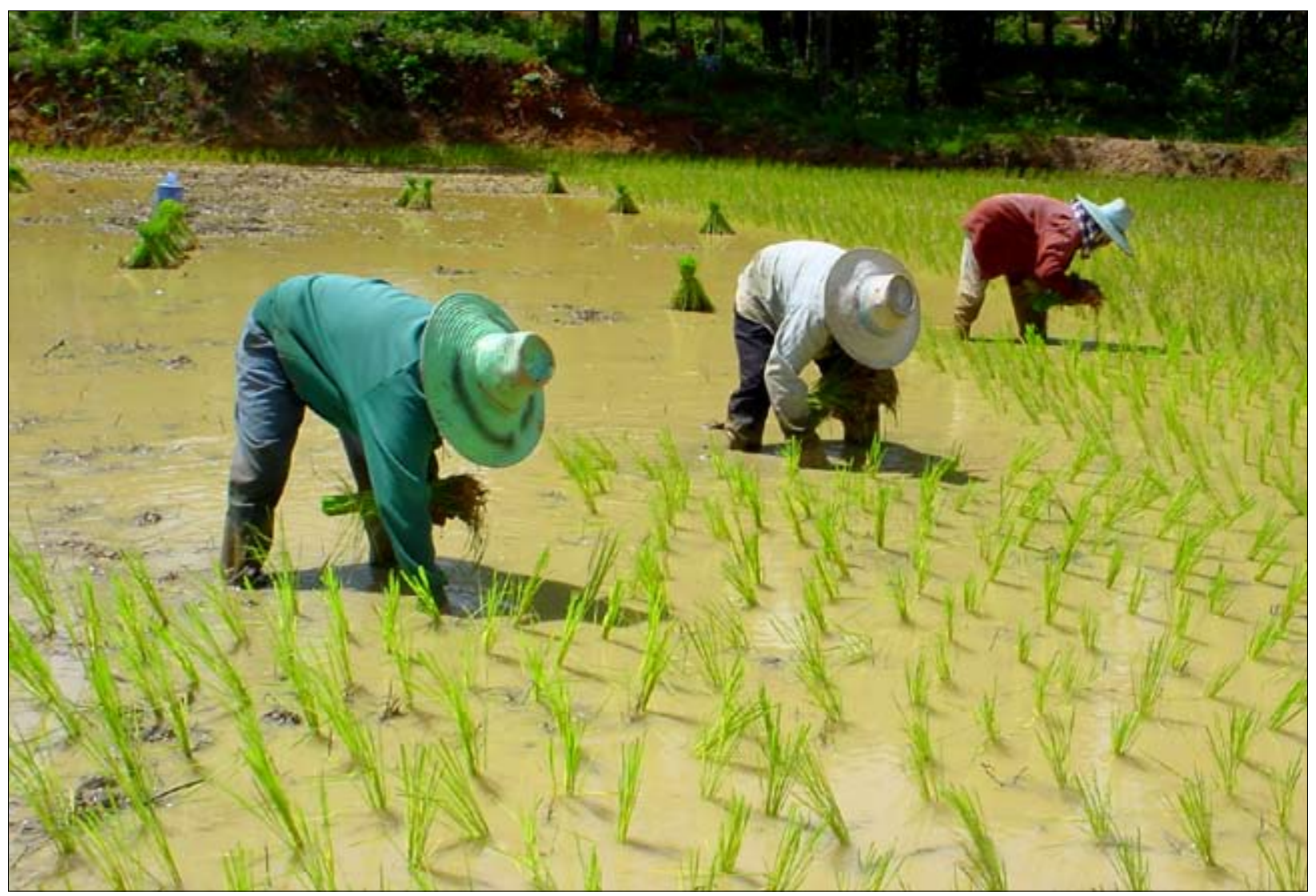

Figure 6. Close-up view of planting rice seedling clusters into a paddy field (na daam).

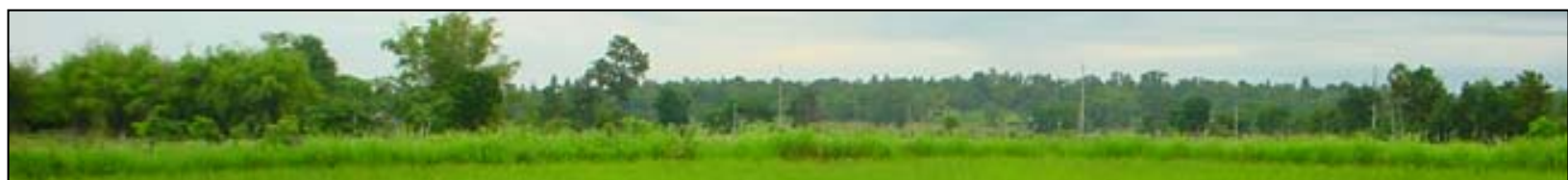

Figure 7. A rice field two months after planting rice seedling clusters. 


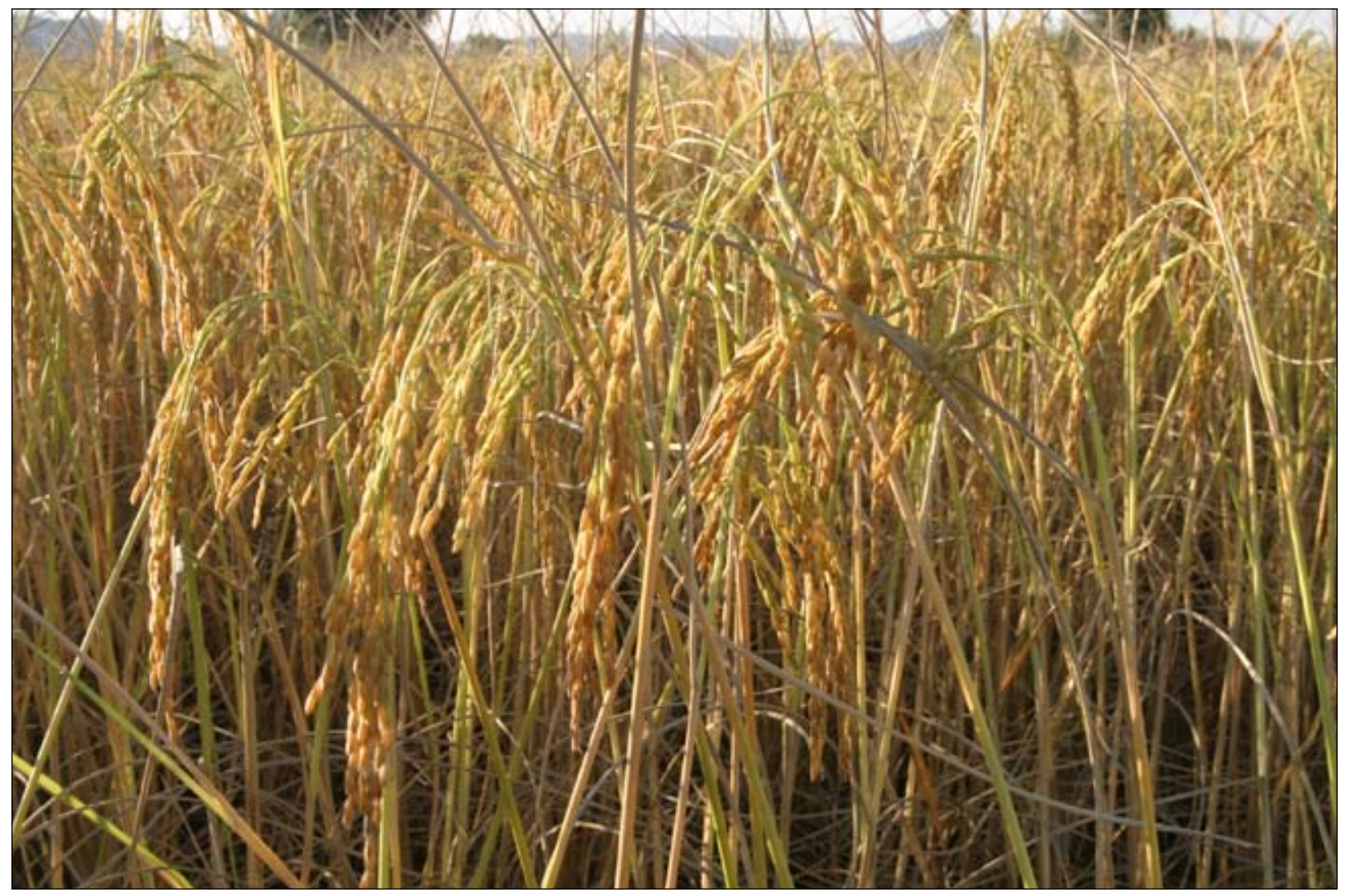

Figure 8. A golden color field of ripening rice fruits.

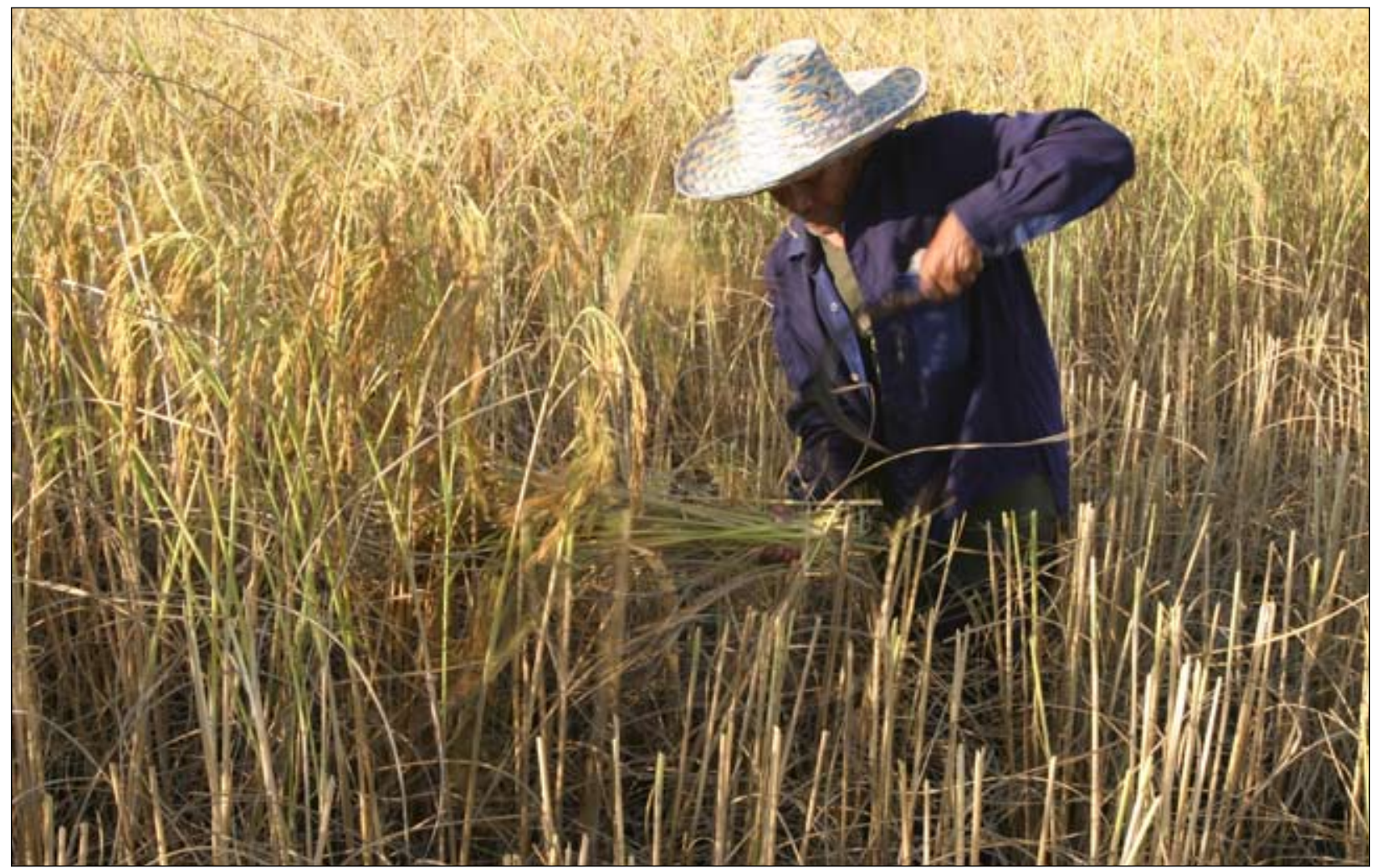

Figure 9. A farmer harvesting ripened rice fruits in the field. 


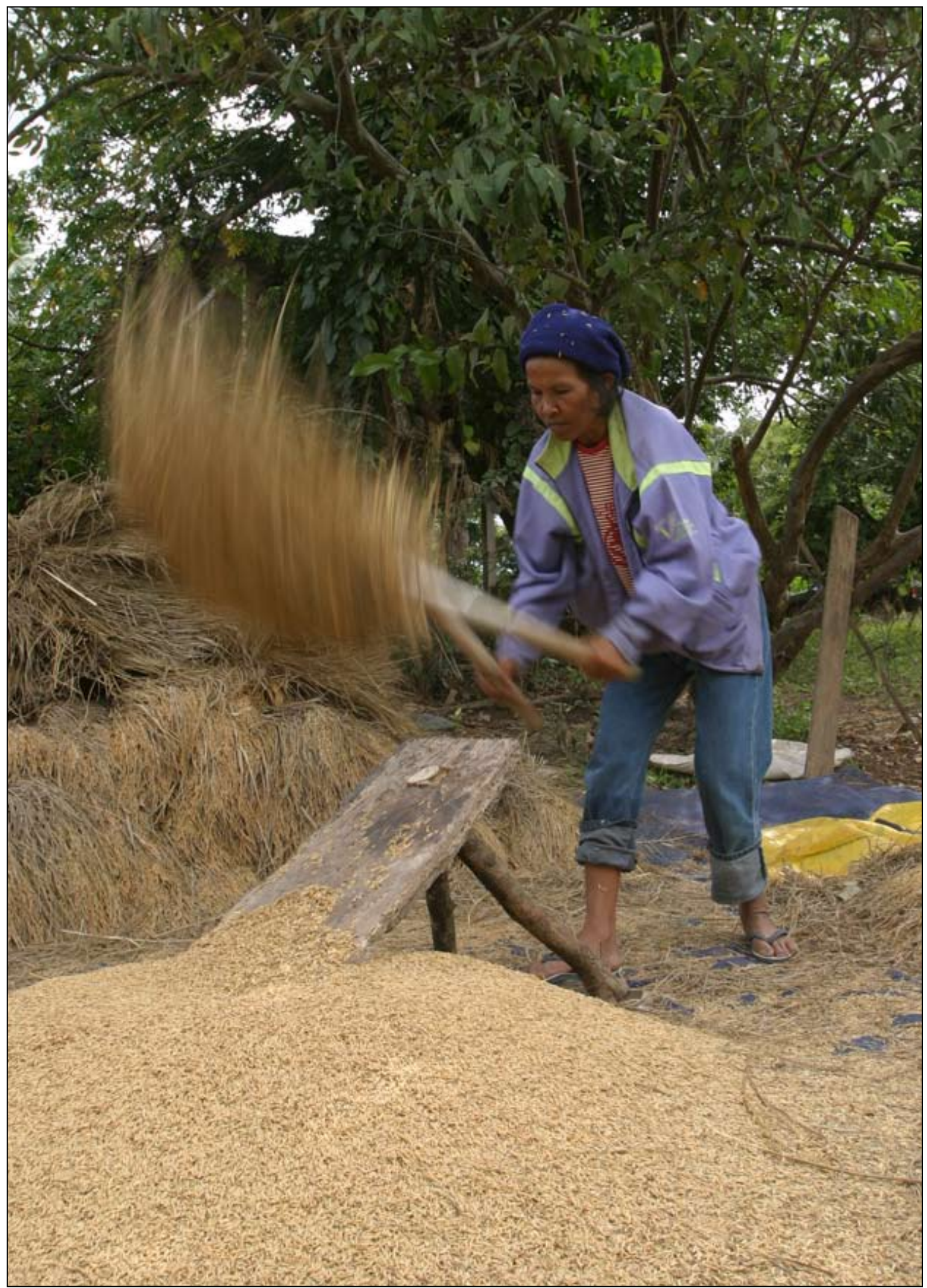

Figure 10. Woman threshing rice. 
Mokkamul - Ethnobotanical Study of Rice Growing in Northeastern, Thailand 221

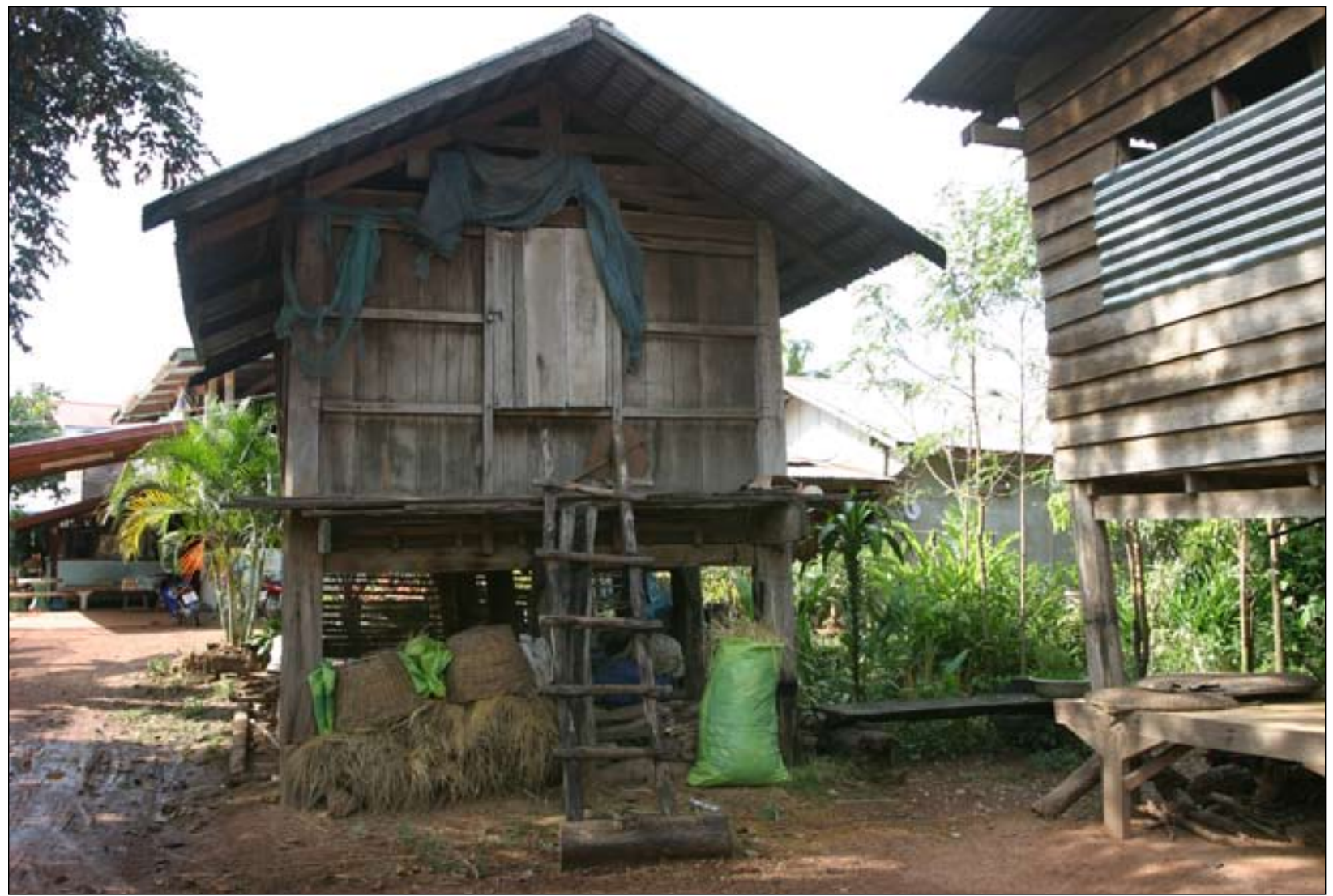

Figure 11. Traditional rice barn for storage of dried rice seeds.

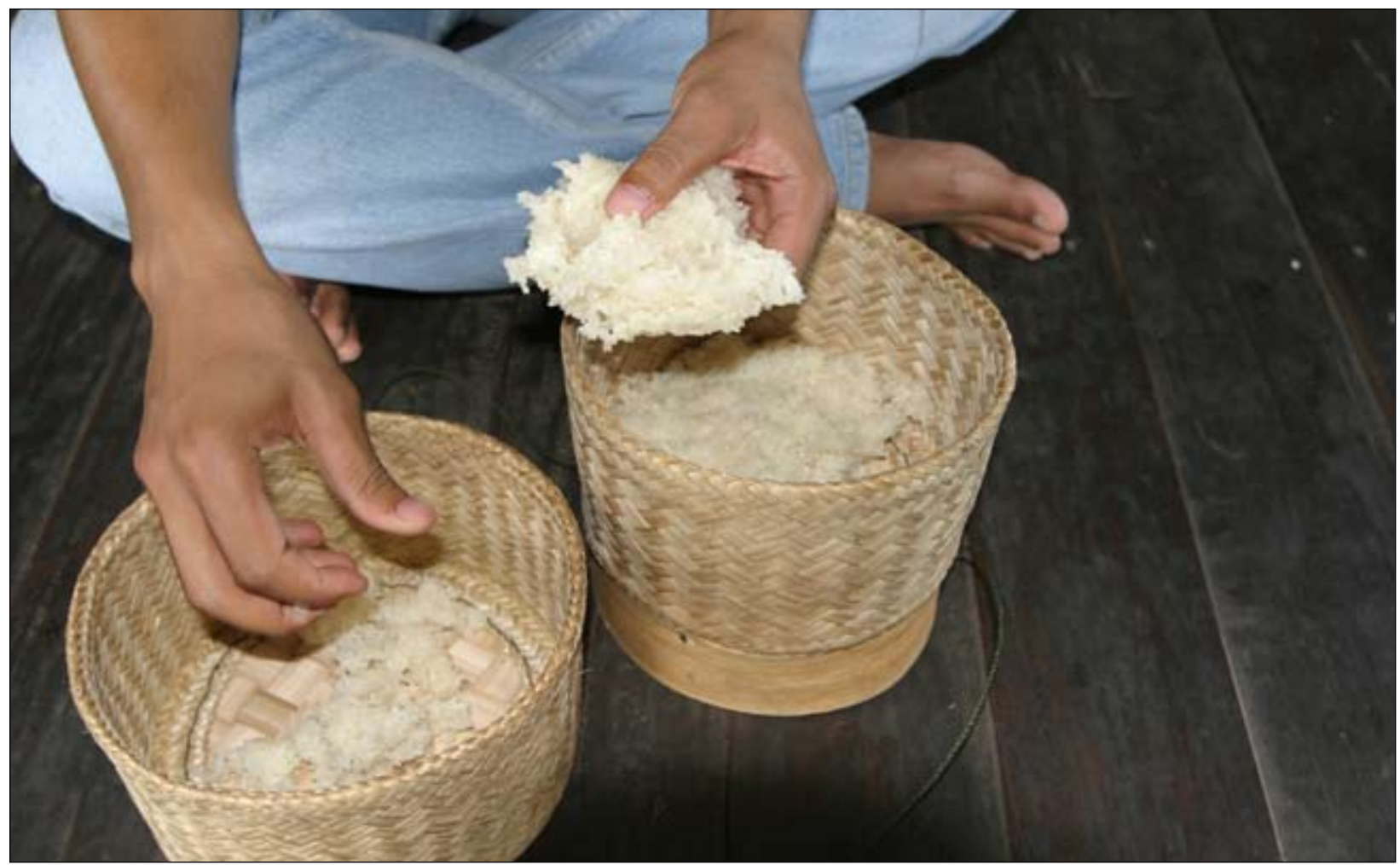

Figure 12. Steamed gluttonous rice ready to eat. 


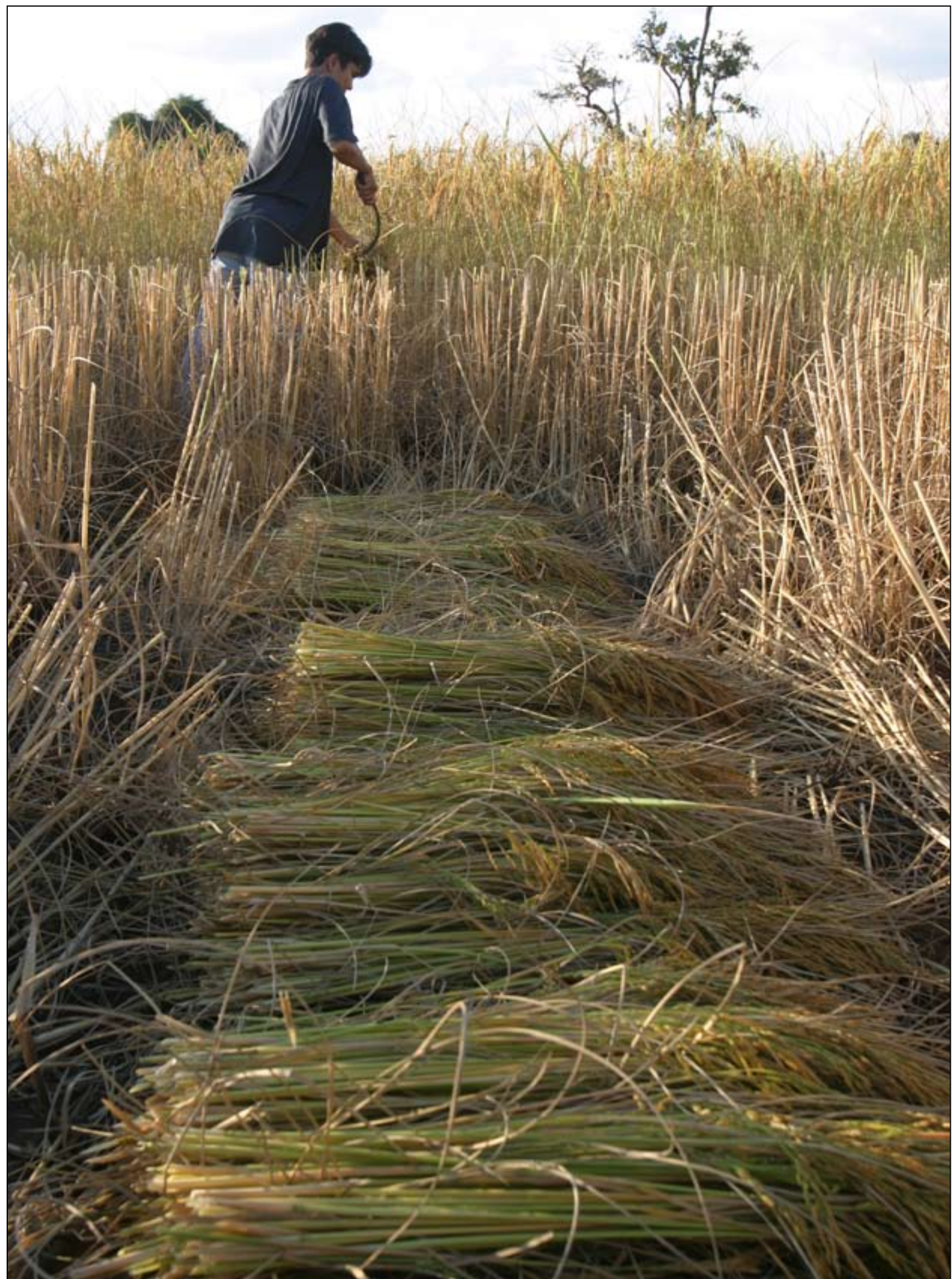

Figure 13. The author harvesting rice in his family's field in Tambon Kham Bo, Waritchaphum, Sakon Nakon, Thailand. 